\title{
Experimental research on dust lifting by propagating shock wave
}

\author{
P. Żydak ${ }^{1} \cdot$ P. Oleszczak ${ }^{1} \cdot$ R. Klemens ${ }^{1}$
}

Received: 6 April 2014 / Revised: 22 February 2016 / Accepted: 20 April 2016 / Published online: 24 May 2016

(C) The Author(s) 2016. This article is published with open access at Springerlink.com

\begin{abstract}
The aim of the presented work was to study the dust lifting process from a layer of dust behind a propagating shock wave. The experiments were conducted with the use of a shock tube and a specially constructed, five-channel laser optical device enabling measurements at five positions located in one vertical plane along the height of the tube. The system enabled measurements of the delay in lifting up of the dust from the layer, and the vertical velocity of the dust cloud was calculated from the dust concentration measurements. The research was carried out for various initial conditions and for three fractions of black coal dust. In the presented tests, three shock wave velocities: 450, 490 and $518 \mathrm{~m} / \mathrm{s}$ and three dust layer thicknesses, equal to $1.0,1.5$ and $2.0 \mathrm{~mm}$, were taken into consideration. On the grounds of the obtained experimental results, it was assumed that the vertical component of the lifted dust velocity is a function of the dust particle diameter, the velocity of the air flow in the channel, the layer thickness and the dust bulk density. It appeared, however, that lifting up of the dust from the thick layers, thicker than $1 \mathrm{~mm}$, is a more complex process than that from thin layers and still requires further research. A possible explanation is that the shock wave action upon the thick layer results in its aggregation in the first stage of the dispersing process, which suppresses the dust lifting process.
\end{abstract}

Keywords Shock waves · Dust lifting · Coal dust

Communicated by K. Kontis and A. Higgins.

R. Klemens

rudolf.klemens@itc.pw.edu.pl

P. Oleszczak

polesz@itc.pw.edu.pl

1 Warsaw University of Technology, Nowowiejska 21/25, 00665 Warsaw, Poland

\section{Introduction}

The experimental research presented in this paper was aimed at investigation of the process of dust lifting from a layer by the flow behind a propagating shock wave. In various industrial facilities and factory buildings, dust layers cover floors, walls, ceilings and various installations. The deposited dust can be quite easily lifted up and dispersed by pressure waves generated by weak explosions, or as a result of damage of compressed gas systems. If the dusts are combustible, an explosive dust-air mixture can be generated, and if it is accidentally ignited, a devastating explosion may occur. Dust mixture explosions constitute one of the major hazards in coal mines that have a high risk of methane explosion, or in other industrial facilities where combustible dusts are present.

The first experimental investigations into the dust lifting process were performed by Gerrard [1]. Later, the process of dust lifting was studied experimentally and theoretically by Boiko [2], Fedorow [3,4], Klemens and Zydak [5-8].

The aim of the work presented here was to study the dust lifting process from relatively thick layers and to determine some parameters needed for development of the model describing the lifting process. The process of dust lifting by the propagating shock wave was recorded with use of three techniques: high-speed shadow pictures, streak pictures and a specially designed laser system.

\section{Research facilities}

The main element of the research facility was a shock tube shown in Fig. 1 [8]. The shock tube has a rectangular shape of $104 \mathrm{~mm} \times 72 \mathrm{~mm}$ cross section. For measuring the dust concentration in the mixture with air, a special, five-channel optical device was constructed. The system is shown in Fig. 2. 


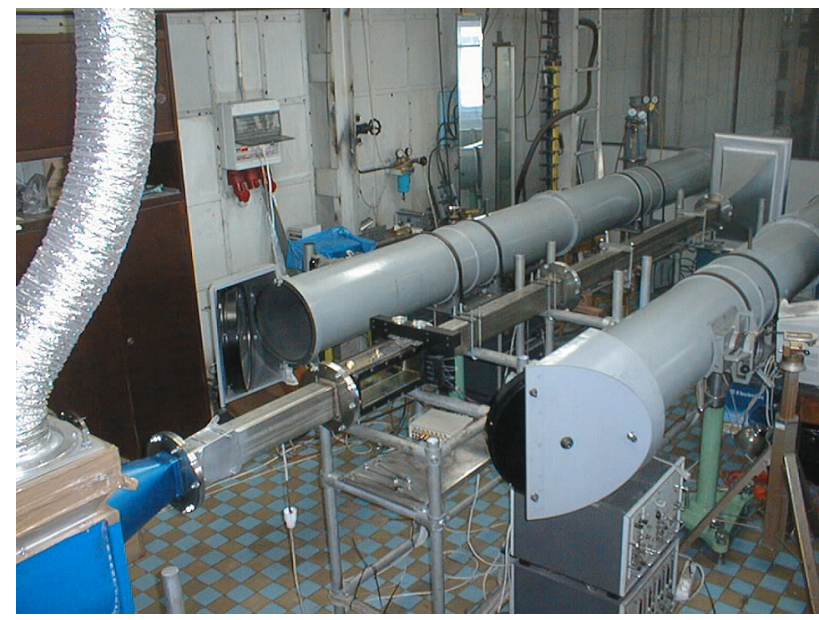

Fig. 1 View of the experimental facility

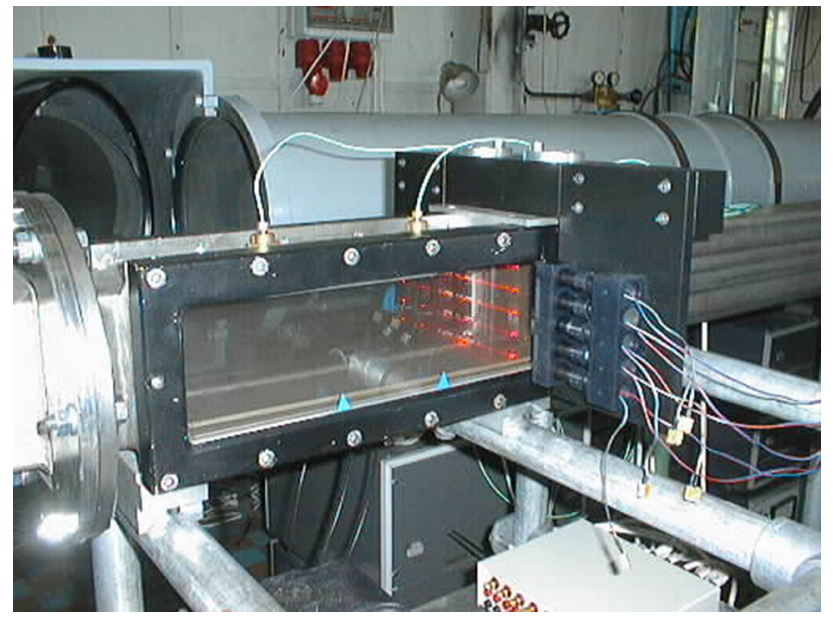

Fig. 2 Dust concentration measurement laser system

It enables measurements of the dust concentration at five points located in one vertical plane along the height of the tube. The system consists of the following elements: laser diodes (TOSHIBA) of $40 \mathrm{~mW}$ power, silicon photodiodes PIN-FDS-100 and a logarithmic amplifier of signals from the photodiodes. The wavelength of the light generated by the laser diodes was equal to $660 \mathrm{~nm}$ and the surface area of the generated laser beam was equal to $13 \mathrm{~mm}^{2}$. The laser diodes of the system were located at five heights above the tube bottom: 8, 29, 50, 71 and $92 \mathrm{~mm}$. The delay in lifting of the dust from the layer behind the propagating shock wave and the vertical velocity of the dust cloud were calculated from the dust concentration measurements, as will be presented in the following sections of the paper. The results obtained with use of the system were compared with those obtained from the analysis of the framing pictures of the process recorded with use of the high-speed digital camera working with the schlieren system and with streak pictures of the dust lifting process.

\section{Coal dust}

The presented research was carried out for three selected coal dusts with various mean diameters of the particles. The first dust, Coal 1, had a mean diameter equal to $18 \mu \mathrm{m}$ and the second dust, Coal 2, had a mean diameter of the particles equal to $80 \mu \mathrm{m}$. The Coal 3 was generated by mixing of Coal 1 and Coal 2 and its mean diameter was equal to $64 \mu \mathrm{m}$. It needs to be emphasized that the majority of the research on the dust lifting process is concerned with monodisperse dusts of a relatively small range of dust particle diameter. In the presented research, polydisperse dust being a mixture of two monodisperse dusts was also investigated. The one-integral volumetric fraction distributions and Rosin-Rammler-Sperling-Bennet distributions for the studied coal dusts were calculated. Three shock wave velocities: 450,490 and $518 \mathrm{~m} / \mathrm{s}$ and three dust layer thicknesses equal to $1.0,1.5$ and $2.0 \mathrm{~mm}$ were considered.

\section{Results of the research}

\subsection{Framing pictures}

To record the process of coal dust lifting by the propagating shock wave, a high-speed digital camera was used. The registration was conducted with a speed equal to 27,000 frames/s and the resolution of the recorded picture was $128 \times 64$ pixels. A single pixel of the picture equals to $2 \mathrm{~mm} \times 2 \mathrm{~mm}$ area. In the observed region of the test section, two indicators were placed. The distance between the indicators equaled $100 \mathrm{~mm}$. The recorded pictures enabled determination of the moment in time when the propagating shock wave reached the first indicator and the moment at which the dust lifting from the layer began. Example pictures recorded are presented in Figs. 3, 4, 5.

From the analysis of the recorded framing pictures, it could be found that initially the dust lifting process is uniform along the length of the dust layer. In the results presented here, the layers were relatively thick. The dust layer was produced by spraying of the dust onto a special plate. The layer was then mechanically smoothened, and in the case of the thicker layers, there was visible roughness of the surface that had an impact on the dust lifting process. In the next stage of the process, lifting of the dust along the entire observed region up to almost half of the height of the tube was very uniform. In the last stage of the process, during which the dust reached the upper part of the tube, some vortices and turbulence of the dust cloud were observed, likely caused by strong turbulence of air flow along the test tube.

On the basis of the recorded pictures, the delay time of the dust lifting was determined. It was defined as the time delay between the moment when the shock wave reached 


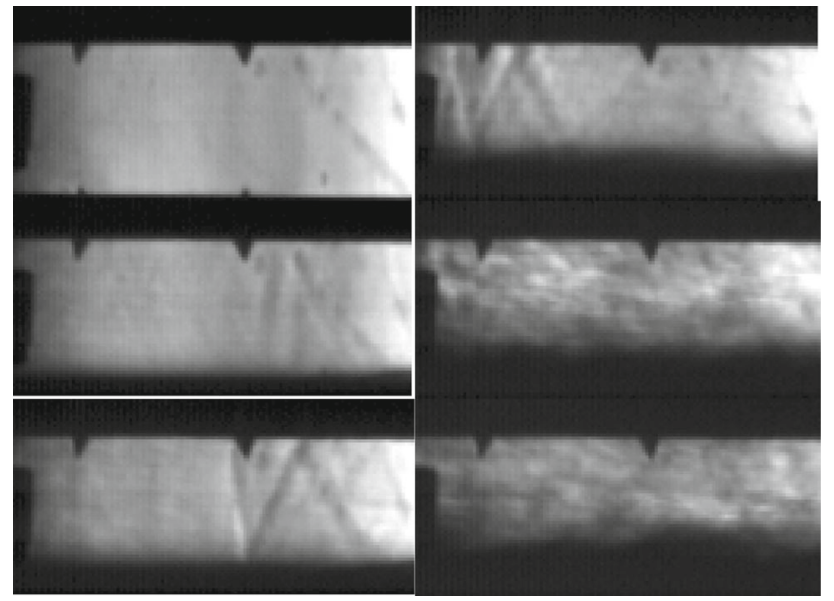

Fig. 3 Framing pictures of coal dust lifting from the layer. Shock wave propagated from the left to the right. Test parameters: coal 2, mean particle diameter equal to $80 \mu \mathrm{m}$, dust layer thickness $1.0 \mathrm{~mm}$, shock wave velocity $518 \mathrm{~m} / \mathrm{s}$, time between frames $1.3 \mathrm{~ms}$

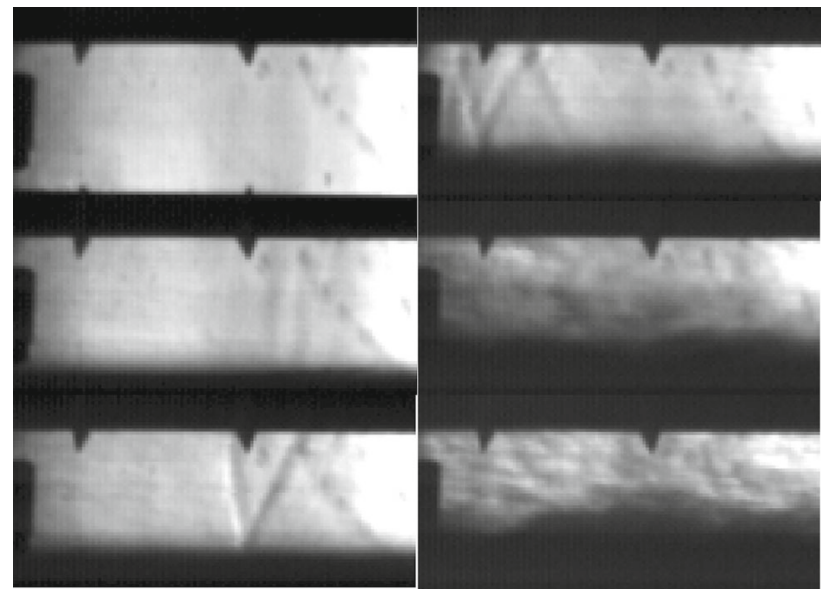

Fig. 4 Framing pictures of coal dust lifting from the layer. Shock wave propagated from the left to the right. Test parameters: coal 2, mean particle diameter equal to $80 \mu \mathrm{m}$, dust layer thickness $1.5 \mathrm{~mm}$, shock wave velocity $518 \mathrm{~m} / \mathrm{s}$, time between frames $1.3 \mathrm{~ms}$

the second indicator and the moment when the dust lifting was first observed. The results are presented on the graph in Fig. 6.

As it can be seen on the graph in Fig. 6, the time of the delay is in the range $0.04-0.14 \mathrm{~ms}$. It can be also found that the influence of the shock wave velocity on the dust lifting delay is similar for all three tested coal dusts and for all three dust layer thicknesses. The increase of the shock wave velocity results in a decrease of the delay time, but the change is not significant. The diameter of the dust particles has a significant influence on the time of the dust lifting delay, especially for lower values of the shock wave velocity. An increase of the diameter of the dust particles results in a decrease of dust lifting delay time. For a shock wave velocity equal to $450 \mathrm{~m} / \mathrm{s}$ and Coal $1(d=18 \mu \mathrm{m})$, the time of dust lifting delay was

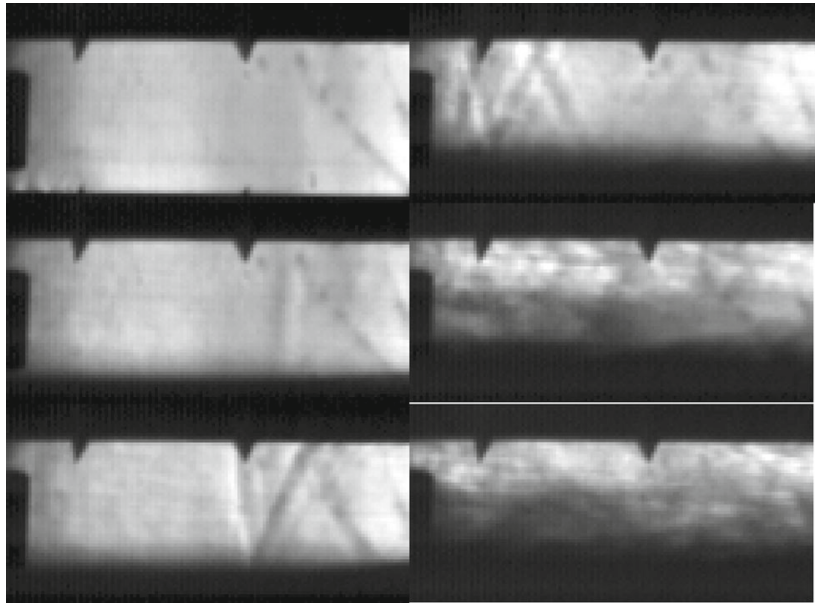

Fig. 5 Framing pictures of coal dust lifting from the layer. Shock wave propagated from the left to the right. Test parameters: coal 2, mean particle diameter equal to $80 \mu \mathrm{m}$, dust layer thickness $2.0 \mathrm{~mm}$, shock wave velocity $518 \mathrm{~m} / \mathrm{s}$, time between frames $1.3 \mathrm{~ms}$

in the range $0.07-0.14 \mathrm{~ms}$, depending on the dust layer thickness and, for Coal $2(d=80 \mu \mathrm{m})$, the time of delay equaled $0.05-0.08 \mathrm{~ms}$, also depending on the dust layer thickness. In general, the increase of the dust layer thickness causes an increase of the delay of the dust lifting, but that influence depends on the velocity and mean diameter of the dust particles.

The results were compared with values obtained with use of a formula presented by Gerrard [1]:

$\Delta=1.7 \cdot \delta^{0.5} \cdot M_{2}^{2}$

where $\Delta$ is the dust lifting delay distance behind the shock wave $(\mathrm{cm}), \delta$ is the thickness of the dust layer $(\mathrm{cm})$, and $M_{2}$ is the Mach number of the flow behind the shock wave relative to the shock wave.

A comparison of the test results with values obtained from (1) is shown in Fig 7. It was found that the formula for delay of the dust lifting (1) cannot be applied here. The values of the delay distance are quite different, but with the same general trend. This discrepancy may be caused by different dust properties and different preparation of the dust layer as well as the measurement techniques used.

\subsection{Streak pictures}

In the experiments, streak pictures of the dust lifting process were also taken using the fast drum camera technique. Examples of the obtained results are presented in Fig. 8. The black dotted line in the pictures indicates the position of the shock wave and two dotted red lines indicate the time when the $6 \mathrm{~mm}$ gap located along the visualization window above the layer is fully covered by the dust mixture. The width of the 
Fig. 6 Dust lifting delay in function of shock wave velocity and dust layer thickness, as obtained from framing pictures
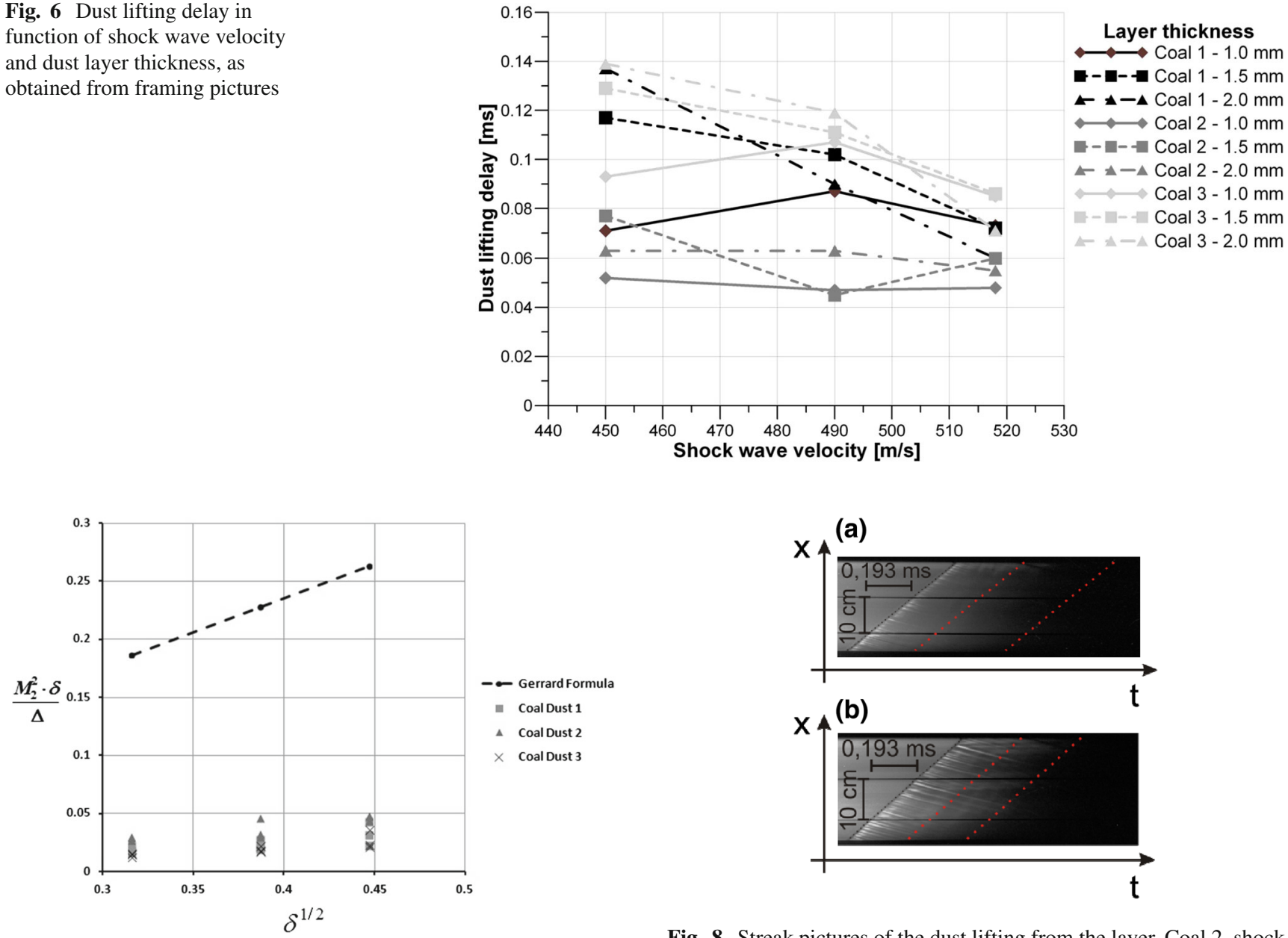

Fig. 7 Comparison of the dust lifting delay measured in the experiments with values obtained with use of Gerrard formula. $\Delta$-Delay of lifting behind the shock wave $(\mathrm{cm}), \delta$ - thickness of the dust layer $(\mathrm{cm})$, $\mathrm{M}_{2}$-Mach number of the flow behind the shock wave relative to the shock wave

schlieren system gap (equal to $6 \mathrm{~mm}$ ) enabled high quality pictures to be obtained with use of the available laboratory equipment.

In Fig. 8, it can be seen that the dust lifting starts just after passage of the propagating shock wave. It is likely that peaks of the layer unevenness were torn off at first. The lefthand side dotted red line indicates a moment of time when the lifted dust reaches a height of $6 \mathrm{~mm}$, i.e., the height of the system gap. The time equals $0.28 \mathrm{~ms}$ for the picture in Fig. 8a and $0.24 \mathrm{~ms}$ for the picture in Fig. 8b. The dotted red line on the right-hand side indicates the moment when the dust concentration at a height equal to $6 \mathrm{~mm}$ reaches its maximal value. This time is much longer than that indicated by the first dotted line on the left-hand side.

The recorded streak pictures clearly show that for the studied layers, the dust lifting process starts just after the propagating shock wave, and the lifted dust reaches the higher parts of the channel with some significant delay.

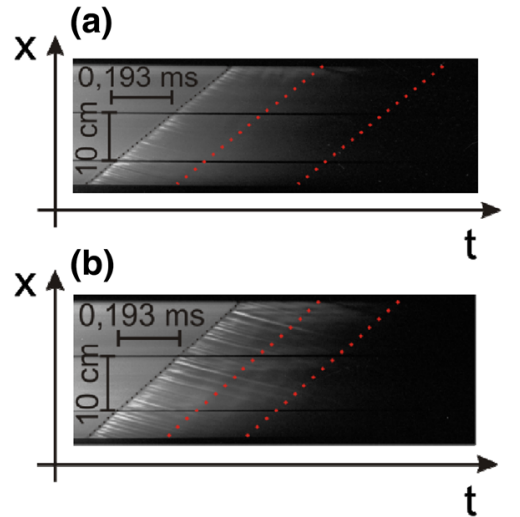

Fig. 8 Streak pictures of the dust lifting from the layer. Coal 2, shock wave velocity $518 \mathrm{~m} / \mathrm{s}$. Black dotted line denotes the shock wave, red dotted lines denote the time when lifted dust filled $6 \mathrm{~mm}$ of the tube height above the dust layer. Layer thickness a $1.0 \mathrm{~mm}$, b $2.0 \mathrm{~mm}$

\subsection{Laser system}

The results of the tests conducted with use of the laser system are presented on the graphs in Figs. 9, 10, 11, 12. Each of the points represents an average of results from 3 to 5 tests conducted for the same dust, dust layer thickness and the same shock wave parameters. The first graph, presented in Fig. 9, shows the time when the dust cloud was lifted to the height of the first laser, $8 \mathrm{~mm}$ above the bottom of the tube. The positions of the lasers were the same for all conducted tests, and the difference in the distance between the first laser and the surface of the layer for various layer thicknesses was taken into account in calculations. Comparing the graph with those presented in Fig. 6 shows that the results obtained with use of the laser system are partially comparable with those from the tests with registration of the framing pictures. The time of delay obtained from analysis of the laser system tests is longer, but other conclusions are very similar. The difference in the delay time originates from the fact 
Fig. 9 Dust lifting delay as a function of shock wave velocity and dust layer thickness, as determined via laser

Fig. 10 Vertical velocity of the dust cloud as a function of shock wave velocity, dust layer thickness and the height range (coal 1)
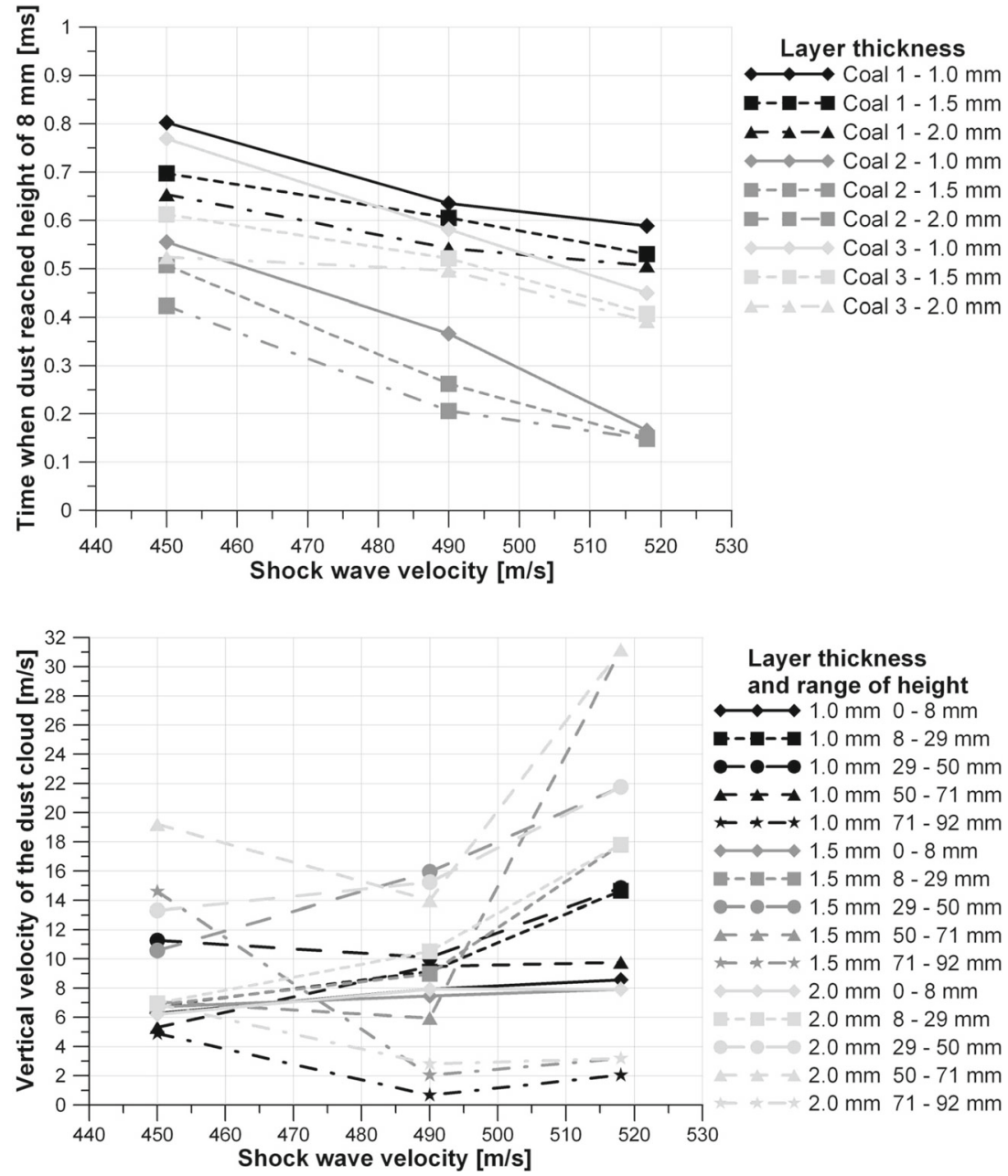

that, in the case of the framing pictures, the beginning of the dust lifting was registered just above the dust layer and, in the case of use of the laser system, the dust cloud was registered when it reached a height of $8 \mathrm{~mm}$ above the test section bottom.

Before these experiments were conducted, the laser system was calibrated to measure dust concentration in air. It was assumed that the dust cloud reached the given laser beam height when the measured dust concentration exceeded $0.02 \mathrm{~kg} / \mathrm{m}^{3}$. The graphs in Figs. 10, 11, 12 present vertical velocities of the dust cloud as a function of the shock wave velocity, the dust layer thickness and the height range, calculated on the basis of the signals from the laser system. The vertical velocities of the dust cloud presented here are average values, calculated using the distance between the laser beams and time between lifting of the dust cloud to the height of the beams. The frequency of sampling of the laser signals was $10 \mathrm{MHz}$.

For Coal 1 (Fig. 10), the maximum vertical velocities were observed for the thickest dust layer and in the range of height between 29 and $71 \mathrm{~mm}$. It can be concluded that up to $71 \mathrm{~mm}$, the dust cloud was accelerated in the vertical direction, and above $71 \mathrm{~mm}$, it was decelerated. The increase of the shock wave velocity and the increase of the layer thickness both caused an increase of the vertical velocity of the dust cloud, but the layer thickness had only a small influence on the results, especially in the range of height $0-8 \mathrm{~mm}$.

The conclusions from the results obtained for Coal 2 (Fig. 11) are slightly different. The increase of the shock wave velocity and the increase of the layer thickness both caused an increase of the vertical velocity of the dust cloud, but in the range of the height $0-8 \mathrm{~mm}$, the layer thickness had only limited influence on the velocity, like in the previous case. But other conclusions are different. In the case of Coal 2, for all three tested thicknesses of the dust layer, the dust cloud had the highest vertical velocity in the range of the height $0-8 \mathrm{~mm}$. Above $8 \mathrm{~mm}$, the cloud was decelerating in the vertical direction.

The results for Coal 3 (Fig. 12), being a mixture of the two previously described coal dusts, are closer to those obtained for Coal 1 . The maximal velocity was observed in the height ranging from 29 to $71 \mathrm{~mm}$, but in this case the results obtained 
Fig. 11 Vertical velocity of the wave velocity, dust layer thickness and the height range (coal 2)

Fig. 12 Vertical velocity of the dust cloud as a function of shock wave velocity, dust layer thickness and the height range (coal 3) dust cloud as a function of shock
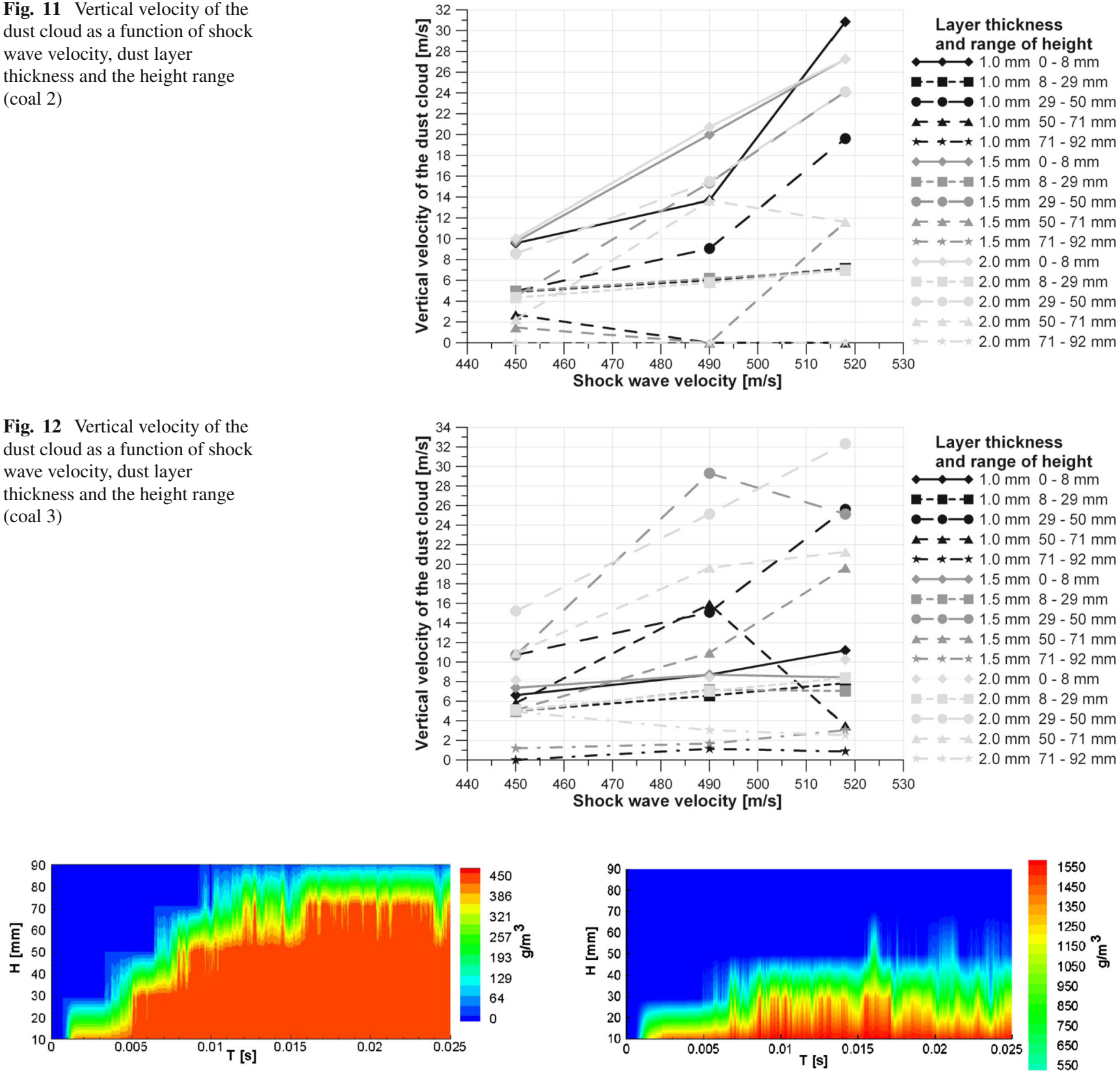

Fig. 13 Distribution of coal dust (coal 1) in the laser system location. Time $0 \mathrm{~s}$ indicates moment when the shock wave propagated through the laser system. Layer thickness $1.0 \mathrm{~mm}$, shock wave velocity $490 \mathrm{~m} / \mathrm{s}$

for a layer thickness equal to $1.0 \mathrm{~mm}$ are more different than those obtained for greater layer thicknesses.

\subsection{Measurements of dust concentration}

The measurements of the lifted dust concentrations were conducted with use of the previously described five-channel laser system. The system was calibrated before the experiments. Example results of the tests are presented in Figs. 13, 14, 15. Concentration of Coal 1 lifted from a 1.0-mm-thick layer by a
Fig. 14 Distribution of coal dust (coal 2) in the laser system location. Time $0 \mathrm{~s}$ indicates moment when the shock wave propagated through the laser system. Layer thickness $1.0 \mathrm{~mm}$, shock wave velocity $490 \mathrm{~m} / \mathrm{s}$

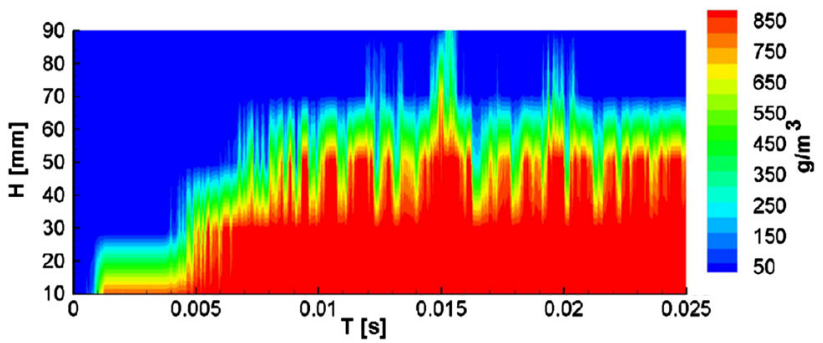

Fig. 15 Distribution of coal dust (coal 3) in the laser system location. Time $0 \mathrm{~s}$ indicates moment when the shock wave propagated through the laser system. Layer thickness $1.0 \mathrm{~mm}$, shock wave velocity $490 \mathrm{~m} / \mathrm{s}$ 
shock wave of velocity of $490 \mathrm{~m} / \mathrm{s}$ as a function of time is presented in Fig. 13. In this case, the cloud of lifted dust reached the top of the channel. Figure 14 presents concentration of Coal 2 dust, lifted for the same conditions of experiment. The cloud of Coal 2 dust was lifted to about half the height of the channel. Coal 3, being a mixture of Coal 1 and 2, was lifted almost to the top of the channel and the result is similar to Coal 1, as can be seen in Fig. 15. These results of experiments suggest that the dust lifting process dynamics and filling of the channel with the dust cloud are determined mainly by the finest particles.

\section{Discussion}

Experimental research on the dust lifting by the propagating shock wave was conducted for three coal dusts of the following sizes: 18,80 and $64 \mu \mathrm{m}$. In the experiments, three layer thicknesses: 1.0, 1.5 and $2.0 \mathrm{~mm}$ and three shock wave velocities: 450,490 and $518 \mathrm{~m} / \mathrm{s}$ were studied. The velocity of the air flowing behind the shock wave was equal to 170,240 and $280 \mathrm{~m} / \mathrm{s}$, respectively. The time of the dust lifting delay was determined using three techniques: high-speed framing pictures, streak pictures and a specially constructed laser system. It was found that the streak pictures give information about lifting of the dust immediately after the shock wave propagation, directly on the layer surface and over the height up to $6 \mathrm{~mm}$. The recorded streak pictures proved that the lifting of the dust particles begins just after the shock wave propagation, irrespective of the layer thickness. The process is dependent on the layer surface condition, e.g., its roughness. The layer is never perfectly even and the dust particles from the peaks of the unevenness are lifted as the first ones. The maximal measured concentration of the lifted dust at a height of $6 \mathrm{~mm}$ above the layer was observed after a time from tens to about two hundreds microseconds after passage of the shock wave. This time corresponds to a distance of $2-7 \mathrm{~cm}$ behind the shock wave. The further development of the dust lifting process depends on the layer thickness and the shock wave velocity. An increase of these two quantities results in more intense and faster lifting of the dust.

The high-speed framing technique made it possible to register the dust lifting process along the length of the dust layer, equal to $0.5 \mathrm{~m}$. However, it did not enable precise determination of the beginning of the dust lifting process. Analysis of the framing pictures showed that the process of dust lifting up to approximately half of the channel height is quite regular and steady. Only when the dust is above this height do vortices and turbulence appear, probably caused by a change of the structure of the flow, which begins to slow down at some distance behind the shock wave. It was also found that the dust of finer particles was lifted higher than the more coarse particles.
The laser system enabled determination of the dust lifting delay time at the height of the location of the first laser, equal to $8 \mathrm{~mm}$. It was found that increase of the shock wave velocity and increase of the layer thickness cause a decrease in the delay time. The increase of the shock wave velocity usually also results in an increase in the height and the dust lifting velocity from the layer. It was observed that in the first stage of the process, the dust of coarse particles is lifted fastest. The time of the dust lifting delay for the tested dusts at the height $8 \mathrm{~mm}$ is in the range $0.1-0.8 \mathrm{~ms}$, which corresponds to $5-27 \mathrm{~cm}$ behind the shock wave.

The vertical component of the lifted dust cloud was also calculated. Unequivocal analysis of the results is rather difficult. It can be found that the vertical velocity increases along with increase of the shock wave velocity and the layer thickness. This trend is especially visible in the lower part of the channel. With the increase of the height, the dust concentration was found to decrease, and it is more difficult to explain the signals registered by the laser system. Therefore, the errors in the results obtained for the upper part of the channel are greater. The influence of the diameter of the dust particles on the vertical velocity for the coal dust is relatively weak. The vertical velocity of the lifted coal dust, as determined in the presented study, was in the range from several to thirty meters per second, depending on the conditions of the particular experiment.

The knowledge of the vertical velocity values was used in the development of a new numerical code, which enables computer simulations of the dust lifting process behind shock waves in industrial facilities. The code was then utilized in a newly elaborated computer program, "DESC", simulating propagation of the dust explosions in industrial facilities. In the program, the dust lifting process is modeled by dust "injection" from the dust layer. The dust injection approach simplifies significantly the modeling of the dust lifting process. The vertical velocity of the injected dust is calculated using (2), presented by Klemens [8].

$v_{2}=16 \cdot 10^{-6} \cdot \delta^{0.0395} \cdot u_{1}^{1.866} \cdot d^{0.1477} \cdot \rho_{\mathrm{m}}^{0.4}\left(\frac{\mathrm{m}}{\mathrm{s}}\right)$

where $\delta$ is the layer thickness $(\mathrm{mm}), u_{1}$ is the horizontal (parallel to dust layer) component of the gas phase velocity $(\mathrm{m} / \mathrm{s}), d$ is the average particle diameter $\left(10^{-6} \mathrm{~m}\right), \rho_{\mathrm{m}}$ is the dust bulk density $\left(\mathrm{kg} / \mathrm{m}^{3}\right), A$ is the empirical parameter, for coal dust equal to 1.2 .

The laser system also enabled the distributions of the dust concentrations to be measured as a function of time over the cross section at the location of the lasers. It was found that for fine particles of diameter equal to $18 \mu \mathrm{m}$, the concentration distributions in the laser cross section are very similar and are almost independent from the layer thickness and the shock wave velocity. The dust is lifted up practically to the 
top wall of the channel. For coarse dust particles of diameter equal to $80 \mu \mathrm{m}$, the maximal height of the dust cloud is significantly lower and strongly depends on the layer thickness and the shock wave velocity. For the case of thicker layers and greater shock wave velocities, the maximum height that the dust cloud is lifted to is found to be greater. The strongly polydisperse dust, Coal 3, being the mixture of Coal 1 and Coal 2, gives results between the ones obtained for Coal 1 and 2 . The dust cloud reaches the height higher than that for Coal $2(80 \mu \mathrm{m})$ and the height is increasing with an increase of the layer thickness and of the shock wave velocity. This result is a very important observation, because it proves that in case of polydisperse dust, the finest fractions of the dust determine the dynamics of the dust lifting and of the velocity of the dust-air mixture generation. Because of this effect, the finest fractions generate the main explosion hazard.

\section{Conclusions}

From the results of this study, the following conclusions can be drawn:

- The beginning of dust lifting from the layer is always observed just behind the propagating shock wave. The further development of the process depends on the layer thickness, the shock wave velocity and dust properties.

- The dust lifting process is uniform along the length of the dust layer.

- Increase of the shock wave velocity and of the layer thickness reduce the delay time of the lifting process and usually results in an increase of the height and velocity of lifting up of the dust from the layer.

- It was observed that in the first stage of the process, the coarse dust is lifted fastest.

- In specific conditions of the dust lifting process, the finest particles are usually lifted to higher heights than coarser particles.

- In the case of polydisperse dusts, the finest fractions determine the mechanism and dynamics of the dust lifting process.
Acknowledgements The work was supported by the Ministry of Science and Higher Education in Poland, Project No. N520 046 31/0719.

Open Access This article is distributed under the terms of the Creative Commons Attribution 4.0 International License (http://creativecomm ons.org/licenses/by/4.0/), which permits unrestricted use, distribution, and reproduction in any medium, provided you give appropriate credit to the original author(s) and the source, provide a link to the Creative Commons license, and indicate if changes were made.

\section{References}

1. Gerrard, J.H.: An experimental investigation of the initial stages of the dispersion of dust by shock waves. Br. J. Appl. Phys. 14(4), 186-192 (1963)

2. Boiko, V.M., Papyrin, A.N.: Dynamics of the formation of a gas suspension behind a shock wave sliding over the surface of a loose material. Combust. Explos. Shock Wave 23(2), 231-235 (1987)

3. Fedorov, A.V., Gosteev, Y.A.: Quantitative description of lifting and ignition of organic fuel dusts in shock wave. J. de Phys. IV (France) 12(7), 89-95 (2002)

4. Fedorov, A.V., Fedorchenko, I.A.: Computation of dust lifting behind a shock wave sliding along the layer. Explos. Shock Wave 41(3), 336-345 (2005)

5. Klemens, R., Wolanski, P., Kosinski, P., Korobeinikov, V.P., Semenov, I.V., Markov, V.V., Menshov, I.S.: On combustion and detonation behind a shock wave propagating over a dust layer. Khimicheskaya Fiz. 20(7), 112-118 (2001)

6. Klemens, R., Zydak, P., Kaluzny, M., Litwin, D., Wolanski, P.: Dynamics of dust dispersion from the layer behind the propagating shock wave. J. Loss Prev. Process Ind. 19, 200-209 (2006)

7. Zydak, P., Klemens, R.: Modelling of dust lifting process behind propagating shock wave. J. Loss Prev. Process Ind. 20, 417-426 (2007)

8. Klemens, R., Oleszczak, P., Zydak, P.: Experimental and numerical investigation into the dynamics of dust lifting up from the layer behind the propagating shock wave. Shock Waves 23(3), 263-270 (2012). doi:10.1007/s00193-012-0376-9

9. Fletcher B.: Propagation of disturbances in granular media, Safety in Mines Research Establishment, SMRE Technical paper pp. 12. Sheffield (1974)

10. Fletcher, B.: The interaction of a shock with a dust deposit. J. Phys. D Appl. Phys. 9(2), 197-202 (1976)

11. Kuhl, A.L., Chien, K.Y., Ferguson, R.E., Glowacki, W.J., Collins, P., Glaz, H.M., Colella, P.: Dust scouring by a turbulent boundary layer behind a shock. Archivum Combust. 9(1/4), 139-147 (1989)

12. Li, Y.C., Harbaugh, A.S., Alexander, C.G., Kauffman, C.W., Sichel, M.: Deflagration to detonation transition fueled by dust layers. Shock Waves 5, 249-258 (1995) 